\title{
General Psychiatry Corpus callosum in schizophrenia with deficit and non-deficit syndrome: a statistical shape analysis
}

\author{
Yașar Türk (10 , ${ }^{1}$ Ilker Ercan, ${ }^{2}$ Ibrahim Sahin, ${ }^{2}$ Basak Erdemli Gursel, ${ }^{3}$ \\ Arda Uzunoglu, ${ }^{2}$ Cem Öge, ${ }^{4}$ Elmas Beyazyüz, ${ }^{5}$ Yakup Albayrak ${ }^{5}$
}

To cite: Türk Y, Ercan I, Sahin I, et al. Corpus callosum in schizophrenia with deficit and non-deficit syndrome: a statistical shape analysis. General Psychiatry 2021;34:e100635. doi:10.1136/ gpsych-2021-100635

Received 06 August 2021 Accepted 27 October 2021

\section{Check for updates}

C Author(s) (or their employer(s)) 2021. Re-use permitted under CC BY-NC. No commercial re-use. See rights and permissions. Published by BMJ.

${ }^{1}$ Department of Radiology, Medical Faculty of Bülent Ecevit University, Kozlu, Zonguldak, Turkey

${ }^{2}$ Department of Biostatistics, Bursa Uludag University Faculty of Medicine, Bursa, Turkey ${ }^{3}$ Department of Radiology, Bursa Uludag University Faculty of Medicine, Bursa, Turkey ${ }^{4}$ Department of Psychiatry, Çorlu State Hospital, Çorlu, Turkey ${ }^{5}$ Department of Psychiatry, Medical Faculty of Tekirdag Namık Kemal University, Tekirdag, Turkey

Correspondence to

Dr Yaşar Türk:

dryasarturk@gmail.com

\section{ABSTRACT}

Background The corpus callosum (CC) is the most targeted region in the cerebrum that integrates cognitive data between homologous areas in the right and left hemispheres.

Aims Our study used statistical analysis to determine whether there was a correlation between shape changes in the CC in patients with schizophrenia (SZ) (deficit syndrome (DS) and non-deficit syndrome (NDS)) and healthy control $(\mathrm{HC})$ subjects.

Methods This study consisted of $27 \mathrm{HC}$ subjects and 50 schizophrenic patients (20 with DS and 30 with NDS). 3 patients with DS and 4 patients with NDS were excluded. Three-dimensional, sagittal, T1-spoiled, gradient-echo imaging was used. Standard anatomical landmarks were selected and marked on each image using specific software.

Results As to comparing the Procrustes mean shapes of the CC, statistically significant differences were observed between HC and SZ (DS+NDS) ( $p=0.017$, James's $\left.F_{i}=73.732\right), H C$ and $D S(p<0.001$, James's $F=140.843)$, HC and NDS ( $p=0.006$, James's $\left.F_{i}=89.178\right)$ and also DS and NDS $(p<0.001$, James's $F=152.967)$. Shape variability in the form of CC was $0.131,0.085,0.082$ and 0.086 in the HC, SZ (DS+NDS), DS and NDS groups, respectively. Conclusions This study reveals callosal shape variations in patients with SZ and their DS and NDS subgroups that take into account the CC's topographic distribution.

\section{INTRODUCTION}

Schizophrenia is a severe psychiatric disease with significant social consequences and has been the subject of decades of neuroimaging study, yet little is known about its neurobiology. ${ }^{1}$ Genetic and histopathological investigations have shown a connection between white matter (WM) genetic variations and genes associated with the risk of schizophrenia. $^{2}{ }^{3}$ Schizophrenia symptoms are thought to be the result of a disruption of the interhemispheric communication. ${ }^{4}$ WM investigations using MRI have supported the interruption theory, showing that the corpus callosum (CC) of patients with schizophrenia (including first-episode schizophrenia) is substantially smaller than that of healthy controls (HC) ${ }^{5-7}$ Despite significant discrepancies in the findings, many investigations using a variety of imaging methods have revealed important details regarding the pathophysiology of schizophrenia. The advent of MRI aided in the identification of the neuroanatomical origins of schizophrenic illness. ${ }^{8}$ Nonetheless, the disease's heterogeneity and the categorisation of all subjects with a diagnosis of 'schizophrenia' have most likely served as a barrier to this invention. Some neuroimaging studies have been aimed at clinical subtypes of schizophrenia. $^{9} \quad 10$ Extensive neuroanatomical, neurobiological and neuropsychological studies have been conducted to delineate possible subgroups of diverse groups of schizophrenia characterised by symptom domain predominance. Carpenter et $a l^{11}$ used the term 'deficit syndrome' (DS) to refer to the presence of main and persistent negative symptoms (a deficit in goal-directed or pleasurable behaviour, voice and non-verbal expression). In patients with DS, impaired cognition, longer course, poorer functional outcomes, lower life quality, and compromised educational and professional activity have all been identified. ${ }^{12}{ }^{13}$ In a diverse population of patients with schizophrenia, the prevalence of DS is about $15 \%$ in firstepisode psychosis and 25\%-30\% overall. ${ }^{14}$ Numerous investigations with limited sample sizes and participant numbers tried to scan the neurostructure of patients with DS and compared the results with those obtained from non-deficit syndrome (NDS) imaging studies. ${ }^{15}$ They found that individuals with DS exhibited lower fractional anisotropy (FA) values in many brain regions compared with those with NDS. This may imply a larger dissociation of the WM in DS. ${ }^{9}{ }^{16-20}$ Voineskos et $a l^{17}$ found that individuals with firstepisode psychosis who also have clinical indications of DS had a greater impairment 
in the WM tract. This demonstrates that disruptions of the interhemispheric communication may contribute to the development of clinical symptoms in DS. Disruption of WM in the superior longitudinal fasciculus and the uncinate fasciculus indicates a disconnection between the temporal, parietal and limbic cortices. ${ }^{9}{ }^{16}$ Spalletta $e t a t^{20}$ and Lei $e t a l^{18}$ both found that the FA values decreased in the CC. Meanwhile, it has been shown that the FA values in the CC splenium and the body have reduced. ${ }^{17} 19$ This indicates that comprehending the psychopathology of DS requires a thorough knowledge of the brain's interhemispheric regions.

Although callosal fibres extend extensively into each cerebral hemisphere, the callosal mid-sagittal border of the cross section has received the greatest attention, both because of the documented correlations between fibre number and callosal area and because it is easier to identify, depict and analyse. ${ }^{21}$ The location of the WM impairment may have psychopathological implications; therefore, we chose to concentrate on determining whether geometric morphometric disturbances in particular areas of the CC may be implicated in the pathophysiology of DS.

Geometric morphometrics is a term that refers to applications of statistical shape analysis based on landmarks. ${ }^{22}$ Geometric morphometrics is a relatively new paradigm for quantitatively analysing the variability and covariance of the forms of biological things. ${ }^{23}{ }^{24}$ Coordinate-based techniques are also known as geometric morphometrics because they preserve all geometric information throughout the data gathering, processing and visualisation processes. ${ }^{25}$

Statistical shape or image analysis, which is used to evaluate two-dimensional or three-dimensional shape data, is gaining increasing popularity in medicine and biology. The principal cause of the increasing use of statistical shape analysis in medicine is technological advances in imaging and a desire to understand the impact of illnesses and environmental variables on organ or organism structure. ${ }^{25}$

To our knowledge, this is the first study to investigate the topographic distribution of the CC in patients with schizophrenia using cranial MRI in order to discover whether the form of the CC varies between schizophrenia and its subgroups (DS and NDS) as well as between HCs.

\section{MATERIALS AND METHODS \\ Subjects}

We recruited $27 \mathrm{HC}$ and 50 patients with schizophrenia who were classified as either DS $(n=20)$ or NDS $(n=30)$ schizophrenia. Patients diagnosed with schizophrenia were enrolled from Çorlu State Hospital and Tekirdağ Namık Kemal University Education and Research Hospital in Turkey. One patient with NDS was unable to finish the imaging test, and the images of 6 patients (3 NDS and 3 DS) had significant artifacts. Thereby, $27 \mathrm{HC}$, 17 DS and 26 NDS were included in the study (figure 1).

The fourth edition of the Diagnostic and Statistical Manual of Mental Disorders (DSM-IV) was used to diagnose schizophrenia. DS was diagnosed using conventional criteria using the Schedule for the Deficit Syndrome, a semistructured interview. ${ }^{26}$ The Turkish version of the Positive and Negative Syndrome Scale (PANSS) was the semistructured interview scale used in this research. The Cronbach's alpha values for the positive syndrome, negative syndrome and overall psychopathology subscales were $0.75,0.77$ and 0.71 on the Turkish version of the PANSS, respectively. As with the original, the Turkish version of the PANSS demonstrated a good level of internal consistency, construct validity and inter-rater reliability. The authors concluded that this adaption is valid and reliable in assessing positive and negative symptoms, as well as general psychopathology, in the Turkish

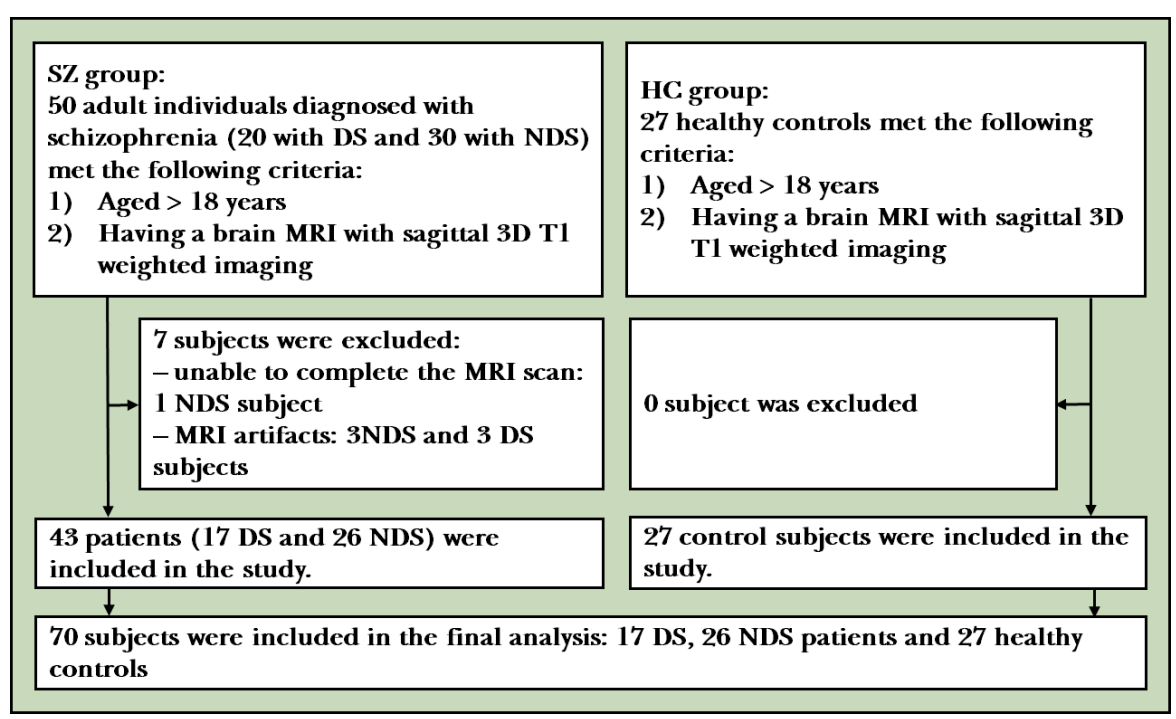

Figure 1 Flowchart for enrolment. 3D, three-dimensional; DS, deficit syndrome; HC, healthy controls; MRI, magnetic resonance imaging; NDS, non-deficit syndrome; SZ, schizophrenia. 
group of patients with schizophrenia. ${ }^{2728}$ All patients with schizophrenia were using at least one antipsychotic medication. All HC participants were tested for a current or lifetime history of DSM-IV disorders using the structured clinical interview for DSM-IV non-patient edition (SCIDNP).$^{29}$ They were also examined to verify that none of their first-degree relatives had ever had psychosis. The study included individuals between the ages of 22 and 68 who met these precise criteria for DS or NDS and were MRI-suitable. Additionally, an HC group of comparable ages and genders was included. Subjects were excluded if they had any of the following criteria: (1) lifelong drug dependence (except psychotic drugs), (2) recent history of drug or alcohol abuse, (3) severe brain injury with loss of consciousness, (4) any significant medical condition or neurological disorder (except schizophrenia) in the schizophrenia (SZ) group, (5) any additional psychological disability or mental retardation or (6) dementia or cognitive impairment according to the DSM-IV.

\section{Acquisition of images}

MRI scans were performed using a GE Brivo 1.5 Tesla machine (General Electric, Milwaukee, Wisconsin, USA) with an eight-channel, sensitivity-encoding head coil. On patients undergoing standard MRI, we conducted inversion recovery in sagittal three-dimensional T1-weighted imaging (field of view (FOV), $240 \mathrm{~mm} \times 240 \mathrm{~mm}$; matrix, $256 \times 256$; segment thickness, $1 \mathrm{~mm}$; Repetition Time (TR) /Time to Echo (TE), $12.2 \mathrm{~ms} / 5.1 \mathrm{~ms}$; flip angle, $\left.15^{\circ}\right)$. Each individual was subjected to the same imaging technique.

\section{Two-dimensional landmark collection}

A single radiologist used TpsDig V.2.30 software to identify and mark the anatomical landmarks. On the CC, 16 anatomical landmarks were described. The 16 landmarks chosen for the CC are as stated in the Sigirli $e t a l^{30}$ report. Figure 2 shows the landmarks used for the CC on an MRI of a subject.

\section{Statistical analysis}

The Shapiro-Wilk test was used to determine if the variables were compatible with a normal distribution. In the case of a normal distribution, $t$ test was used to compare

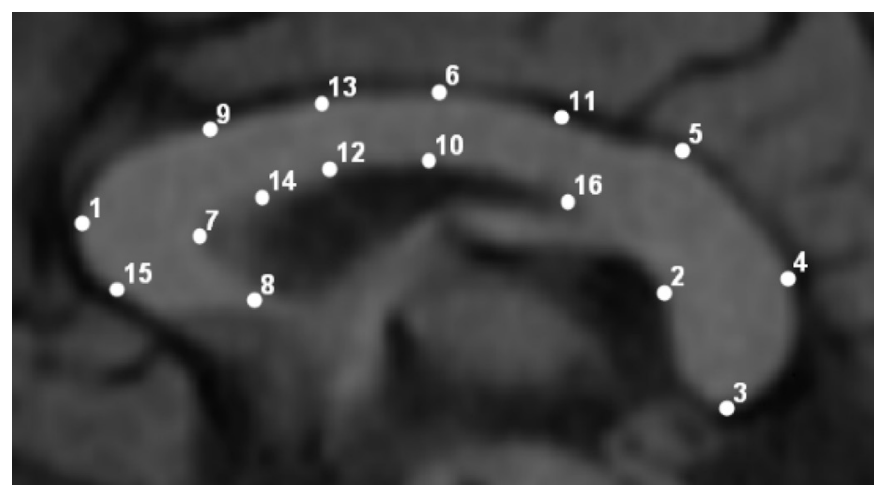

Figure 2 The corpus callosum landmarks are visible on this mid-sagittal slice. the groups and mean (SD) was used as descriptive values. If normal distribution was not provided, comparisons were made using Mann-Whitney $\mathrm{U}$ test, with descriptive values given as median (minimum-maximum). Categorical variables are denoted by n $(\%)$. Pearson's $\chi^{2}$ test was used to compare categorical variables between groups.

For the comparison of shape, Procrustes analysis was used. Box's $M$ test was used to determine if the covariance-variance matrices were homogenous. When the matrices of variance and covariance were homogenous, the Hotelling $\mathrm{T}^{2}$ test was used to compare Procrustes mean shapes, whereas James's $F_{j}$ test was used when the matrices of variance and covariance were not homogenous. To get an overall assessment of shape variability, the root mean square of Kendall's Riemannian distance $r$ to the mean shape was employed.

Thin-plate spline (TPS) analysis was used to evaluate the shape deformations. The Procrustes mean shapes were generated for TPS analysis. The regions with the largest reductions or enlargements were colour-coded to indicate deformations based on the findings of the TPS analysis. R V.3.6.3 ('shapes'), PAST V.2.17 and SPSS V.22 applications were used for statistical analysis in this report. The significance level was established as $\alpha=0.05$.

\section{Landmark reliability}

We used the generalisability theorem (GT) to determine the intrarater reliability coefficient $(G, 0 \leq G \leq 1)$ for a two-facet crossing configuration (landmark pairs-byrater-by-subject). ${ }^{31}$ The GT employs the generalisability coefficient to quantify the dependability of relative (normreferenced) interpretations. ${ }^{32} \mathrm{~A}$ single rater recognised the anatomical landmarks in this study. The reliability of the rater was determined by repeated landmarks on groups. A single investigator gathered landmarks on the $\mathrm{CC}$ and then re-marked the same locations on the same 20 participants after a month (10 cases and $10 \mathrm{HCs})$. The rating indicated high degree of repeatability for patients $(\mathrm{G}=0.9979)$, controls $(\mathrm{G}=0.9961)$ and overall $(\mathrm{G}=0.998$ 4). A landmark reliability account was created ${ }^{31}$ at http:/ / biostat.home.uludag.edu.tr/landmark_reliability/G_ coefficient.html.

\section{RESULTS}

\section{Clinical characteristics}

There were no statistically significant differences in age or gender between the HC and patient (DS+NDS) groups or between the DS and NDS groups (table 1).

In DS, Mini-Mental State Exam (MMSE), PANSS positive, PANSS negative, schizophrenia onset age and diagnosis time were 24.53 (2.90), 11 (6-47), 77 (19-108), 20 (14-36) and 13.11 (9.93), respectively.

In NDS, MMSE, PANSS positive, PANSS negative, schizophrenia onset age and diagnosis time were 25.32 (2.87), 25 (2-104), 21 (0-101), $22(15-59)$ and 13.05 (10.99), respectively. 
Table 1 Demographics of the study groups

\begin{tabular}{|c|c|c|c|c|c|c|c|}
\hline & HC $(n=27)$ & DS+NDS $(n=43)$ & DS (n=17) & NDS $(n=26)$ & Comparison of groups & $\begin{array}{l}\text { Test statistics } \\
\text { Mann-Whitney } U \text { test } / \chi^{2}\end{array}$ & $P$ value \\
\hline \multirow[t]{4}{*}{ Age (years) } & $29(24-68)$ & $39(22-67)$ & $39(22-67)$ & $38.5(22-66)$ & HC-SZ & $431.0^{\star}$ & 0.071 \\
\hline & & & & & HC-DS & $153.5^{\star}$ & 0.066 \\
\hline & & & & & HC-NDS & $277.5^{*}$ & 0.190 \\
\hline & & & & & DS-NDS & $216.0^{*}$ & 0.901 \\
\hline \multirow[t]{3}{*}{ Gender (male) } & 16 (59.26) & 27 (62.79) & 9 (52.94) & $18(69.23)$ & HC-SZ & $0.002 \dagger$ & 0.966 \\
\hline & & & & & HC-NDS & $0.221 \dagger$ & 0.638 \\
\hline & & & & & DS-NDS & $0.574 \dagger$ & 0.449 \\
\hline
\end{tabular}

Data are given as $\mathrm{n}(\%)$ or median (minimum-maximum)

*Mann-Whitney $\mathrm{U}$ test statistics.

$\dagger \chi^{2}$ test statistics.

DS, deficit syndrome; HC, healthy control; NDS, non-deficit syndrome; SZ, schizophrenia (DS+NDS).

\section{Statistical shape analysis}

According to the CC shapes, there were statistically significant differences between the HC and SZ (DS+NDS) groups ( $\mathrm{p}=0.017$, James's $\mathrm{F}=73.732)$, the $\mathrm{HC}$ and the DS groups ( $\mathrm{p}<0.001$, James's $\mathrm{F}=140.843$ ) and the $\mathrm{HC}$ and the NDS groups $\left(\mathrm{p}=0.006\right.$, James's $\left.\mathrm{F}_{\mathrm{j}}=89.178\right)$, as well as between the DS and the NDS groups $(p<0.001$, James's $\left.\mathrm{F}_{\mathrm{j}}=152.967\right)$.

The shape variability in the form of the $\mathrm{CC}$ in the $\mathrm{HC}$, patient (DS+NDS), DS and NDS groups was 0.131, 0.085, 0.082 and 0.086 , respectively. The CC shape variability in the HC group was higher than that of patients and their subgroups. The variability in patients and their subgroups was similar.

According to the CC deformation of the HC group compared with the patient (DS+NDS) group, the area between landmarks 1, 15 and 7 (genu of the CC), the region between landmarks 6 and 11 (on the superior boundary of the region between the posterior body and the isthmus of the CC), and the region between landmarks 2, 4, 5 and 16 (between the isthmus and the splenium of the CC) were found to have the biggest deformation.

With the exception of landmark 16, the greatest deformation observed in HC to patients with DS was identical to that seen in HC to individuals with schizophrenia.

Per the HC group's CC deformation in comparison with NDS, the greatest deformation was seen between landmarks 1, 15 and 7 (genu of the CC), between landmarks 12 and 13 (between the anterior and posterior bodies of the CC) and between landmarks 2, 3, 4, 5, 11 and 16 (the isthmus and the splenium of the CC).

When the shape of the CC was examined from a nondeficit to a deficit state, a high degree of deformation was observed in the regions between landmarks 1, 15, 7 and 8 (rostrum) and in the region between landmarks 4 and 5 (superior zone of the region between the CC's isthmus and splenium). Furthermore, when comparing DS and NDS with HC using the CC shape analysis, the DS group had greater shape distortion. Graphs are given as two dimensions (abscissa $(\mathrm{x})$ and ordinate $(\mathrm{y})$ ) in figure 3 . The expansion and contraction factors at the landmarks were numerically displayed in TPS analysis. In TPS charts, the expansion factor was defined as the value is greater than one and the contraction factor was defined as the value is less than one. Deformation areas were interpreted according to the colour scale in the TPS graphs on the right of figure 3 .

\section{DISCUSSION}

\section{Main findings}

Numerous brain imaging investigations have shown abnormal anatomical and functional brain integration in individuals with schizophrenia, corroborating the notion that schizophrenia is a disconnection disease. ${ }^{33} 34$ Schizophrenia involves interhemispheric connectivity, which raises concerns about the CC's structural integrity. ${ }^{35} 36$ The CC is the biggest commissural conduit connecting the two hemispheres of the brain. It comprises an organised bundle of nerve fibres of varying thickness that aggregate to create a mass at the midline and spread to topographically similar areas in either hemisphere. It has been extensively studied in order to determine its involvement in hemispheric communication as well as lateralised brain activity and cognition.

The aim of this research was to look at the shape differences and variations in the CC's geometric and morphometric form in patients with schizophrenia, as well as the subgroups of DS and NDS. Also, if there are any subregions of these CC variations, they should be evaluated. We used a landmark-based geometric morphometric technique to assess these changes. In comparison with the $\mathrm{HC}$ group, the results revealed a significant difference in the CC form in patients with both DS and NDS. Furthermore, subregional analyses revealed that the most noticeable changes were seen in the genu of the CC, on the superior border of the region between the posterior body and the isthmus of the CC, and between the isthmus and the splenium of the CC. According to diffusion tractographybased parcellations of the CC along the mid-section and connectional division of the cortex, these regions are associated with prefrontal, cingulate and superior 

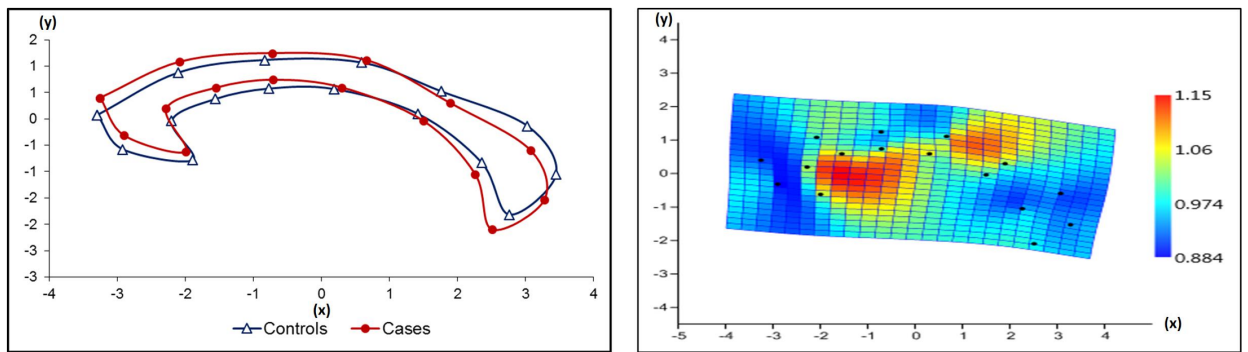

\section{A}
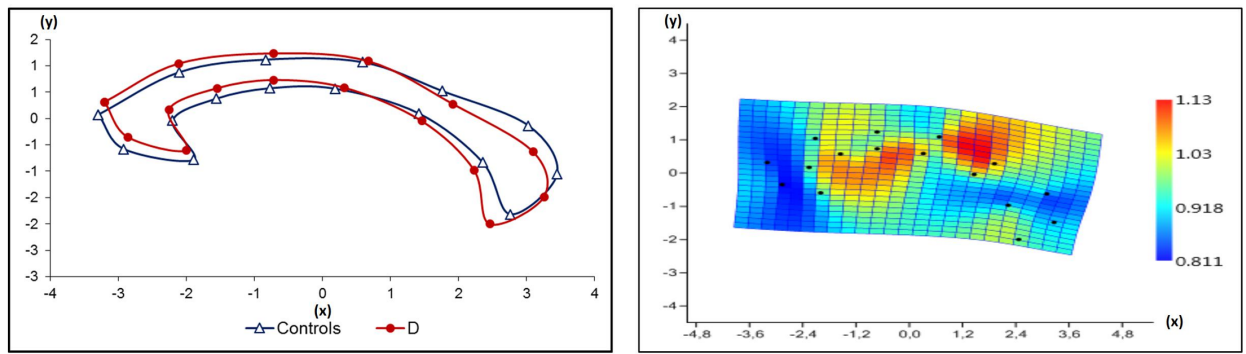

B
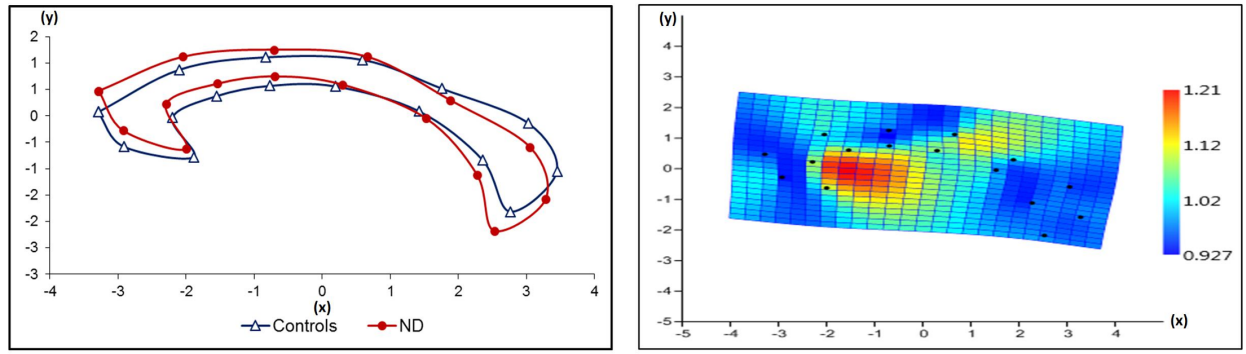

C
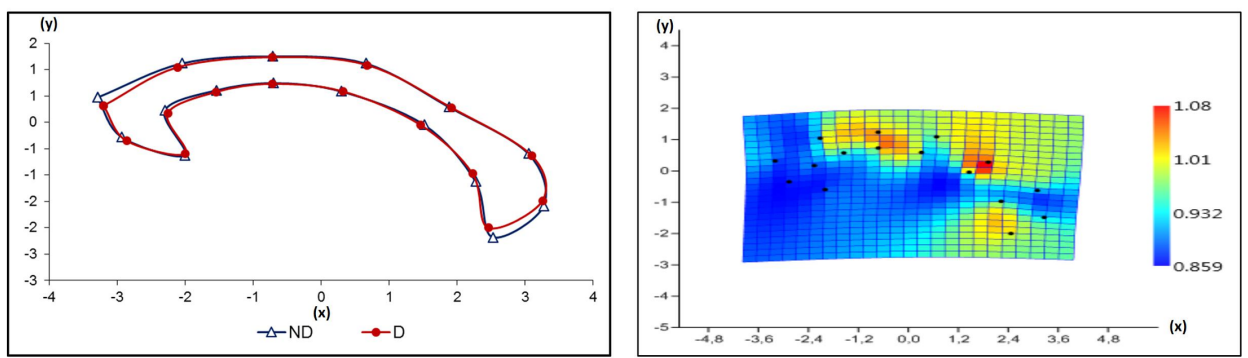

D

Figure 3 Left: (A) For the corpus callosum images of HC and SZ, Procrustes mean shapes were used; (B) images of HC and DS; (C) images of HC and NDS and (D) images of NDS and DS. Right: (A) The average corpus callosum shape deformation from $\mathrm{HC}$ to SZ as depicted by a thin-plate spline; (B) from HC to DS; (C) from HC to NDS and (D) from NDS to DS. D, deficit; DS, deficit syndrome; HC, healthy control; ND, non-deficit; NDS, non-deficit syndrome; SZ, schizophrenia (DS+NDS).

temporal, respectively. ${ }^{37}$ In a diffusion tensor imaging (DTI) analysis, Park et $a l^{38}$ discovered that the genu of the CC incorporates bilateral prefrontal cortex in HC. The prefrontal cortex is heavily involved in working memory and processing speed ${ }^{39}$ Hypoactivation of the dorsolateral prefrontal cortex has been observed in patients with schizophrenia when performing the n-back exercise. ${ }^{40}$ Friston ${ }^{41}$ proposed an irregular functional interaction between the prefrontal and temporal regions in a study of the disconnection theory of schizophrenia. In addition, Kim $e t a t^{42}$ looked at grey matter microstructural defects and working memory deficits in people with schizophrenia and found that the schizophrenia group had significantly higher axial diffusivity (AD), radial diffusivity (RD), and trace (TR) values in various frontal, temporal and anterior cingulate regions than the HC group. Our findings seem to correlate to the fibre tracts of the aforementioned damaged grey matter in both hemispheres, as determined by Catani and Thiebaut de Schotten's ${ }^{37}$ callosum parcellations. Additionally, a variety of CC deformities were identified in the DS group, which may be linked to the tracts of the CC that originate from the 
superior temporal gyrus, based on Catani and Thiebaut de Schotten's ${ }^{37}$ CC parcellations and mid-section connectional division, whereas in the NDS group the shape distortion of the CC was seen in non-specific areas associated with the parietal, occipital and temporal regions. In the absence of significant variations between groups in total brain volume or total cranial volume, Fischer et $a t^{43}$ discovered that DS cases were differentiated from NDS and healthy reference groups by smaller superior frontal and superior and middle temporal gyral volumes. As a result, the deformities they observed are not solely due to a reduction in total brain volume, but are specific to certain cortical regions. In a Region of Interest (ROI) analysis, the DS group had lower left temporal lobe volume than the NDS and stable matching groups. ${ }^{44}$ Decreased grey and white matter signal strength in the superior temporal gyrus in DS compared with a stable reference group has also been found in voxel-based morphometry (VBM) studies. ${ }^{1045} 46$ Numerous neurostructural MRI investigations have revealed abnormalities in the temporal lobes in DS, which seems to corroborate our results.

Our HC group's CC shape variability was higher than that of the patientSZ group and their subgroups, while the variability in SZ group and the variability in their subgroups were comparable, which may indicate a neurodevelopmental abnormality that leads to fewer changes in CC shape in patients with schizophrenia and their deficit and non-deficit subgroups. Tao $e t a l^{47}$ discovered a robust callosal thickness reduction in schizophrenia, which they conclude is attributable to a brain maturing abnormality that causes reduced functional cohesion and interhemispheric contact integration. The deficit was limited to the back of the mid-section and was related to the severity of negative symptoms.

Joshi et $a l^{21}$ investigated the effect of sex and age on callosal shape abnormalities and disease-associated genetic liability in schizophrenia and showed both global and local impact of age, sex and biological risk on CC shape variation. Specifically, the research found that individual variations in age, sex and illness status were unrelated to callosal size. Despite smaller levels of variance, callosal morphology was similarly linked to schizophrenia and genetic susceptibility to schizophrenia. They discovered that as people get older, the degree of constriction and arching of the CC increases. Despite the large sample size (218), the cohort was diverse, with just 48 people with a DSM-IV diagnosis of schizophrenia participating in the research, and the general schizophrenia groups were examined without distinguishing DS and NDS subgroups. Our report is the first study to use shape analysis and the geometric morphometric technique to examine patients with schizophrenia and compare the DS and NDS subtypes of schizophrenia directly.

The Sigirli $e t a l^{30} 48$ research examined the shape deformation of the CC in patients with restless leg syndrome (RLS) and multiple sclerosis (MS) using the shape analysis technique and found that the most apparent deformation occurred in the posterior mid-body and anterior regions of the CC, respectively. According to connectional division (cortical parcellation based on diffusion tractography), the posterior mid-body region of the CC is linked with motor cortex in RLS, which they hypothesised was owing to myelin insufficiency. Nonetheless, the greatest distortion in the anterior area of the CC in MS patients was owing to the sensitivity to MS damage of the CC's anterior region. These findings bolster the dependability of shape analysis and the geometric morphometric approach, which is compatible with the existing literature on MRI-based research techniques, such as DTI of the WM tracts and VBM.

\section{Limitations}

The limitation of the study could be attributed to its small sample size, particularly in the deficit syndrome group.

\section{Implications}

The observed deformations are most pronounced in the genu of the CC, along the superior border of the region between the posterior body and the isthmus of the CC, and between the isthmus and the splenium of the CC, implying that the development of schizophrenia has an effect on the prefrontal and parietotemporal cortices. Furthermore, the superior zone of the CC between the isthmus, splenium and rostrum was damaged in the DS group, which may be explained by DS development affecting the superior temporal cortex and cingulum. These results suggest that we should go beyond symptomatology in schizophrenia and its subtypes and that tractographical studies should be conducted to identify WM pathways of the CC in a connectome study.

Contributors YT is responsible for the overall content as the guarantor and had complete access to all of the data in the study and also is solely responsible for the integrity of the data and the correctness of the data analysis. YT, IE, IS, BEG and AU contributed to the concept and design. YT, CÖ, EB and YA were responsible for data collection, analysis and interpretation. YT, IE, IS, BEG and AU drafted the manuscript.

Funding The authors have not declared a specific grant for this research from any funding agency in the public, commercial or not-for-profit sectors.

Competing interests None declared.

Patient consent for publication Not required.

Ethics approval This retrospective study was approved by our internal ethics committee (permission granted/decision no: 2020.15.01.15).

Provenance and peer review Not commissioned; externally peer reviewed.

Data availability statement Data are available upon reasonable request.

Open access This is an open access article distributed in accordance with the Creative Commons Attribution Non Commercial (CC BY-NC 4.0) license, which permits others to distribute, remix, adapt, build upon this work non-commercially, and license their derivative works on different terms, provided the original work is properly cited, appropriate credit is given, any changes made indicated, and the use is non-commercial. See: http://creativecommons.org/licenses/by-nc/4.0/.

ORCID iD

Yaşar Türk http://orcid.org/0000-0001-6728-3664

\section{REFERENCES}

1 Wittchen HU, Jacobi F, Rehm J, et al. The size and burden of mental disorders and other disorders of the brain in Europe 2010. Eur Neuropsychopharmacol 2011;21:655-79. 
2 Kelly S, Jahanshad N, Zalesky A, et al. Widespread white matter microstructural differences in schizophrenia across 4322 individuals: results from the ENIGMA Schizophrenia DTI Working Group. Mol Psychiatry 2018;23:1261-9.

3 Bohlken MM, Brouwer RM, Mandl RCW, et al. Genetic variation in schizophrenia liability is shared with intellectual ability and brain structure. Schizophr Bull 2016;42:1167-75.

4 Crow TJ. Schizophrenia as a transcallosal misconnection syndrome. Schizophr Res 1998;30:111-4.

5 Arnone D, McIntosh AM, Tan GMY, et al. Meta-Analysis of magnetic resonance imaging studies of the corpus callosum in schizophrenia. Schizophr Res 2008;101:124-32.

6 Collinson SL, Gan SC, Woon PS, et al. Corpus callosum morphology in first-episode and chronic schizophrenia: combined magnetic resonance and diffusion tensor imaging study of Chinese Singaporean patients. Br J Psychiatry 2014;204:55-60.

7 Keshavan MS, Diwadkar VA, Harenski K, et al. Abnormalities of the corpus callosum in first episode, treatment naive schizophrenia. $J$ Neurol Neurosurg Psychiatry 2002;72:757-60.

8 Shenton ME, Kikinis R, Jolesz FA, et al. Abnormalities of the left temporal lobe and thought disorder in schizophrenia. N Engl J Med Overseas Ed 1992;327:604-12.

9 Rowland LM, Spieker EA, Francis A, et al. White matter alterations in deficit schizophrenia. Neuropsychopharmacology 2009;34:1514-22.

10 Galderisi S, Quarantelli M, Volpe U, et al. Patterns of structural MRI abnormalities in deficit and nondeficit schizophrenia. Schizophr Bull 2008;34:393-401.

11 Carpenter WT, Heinrichs DW, Wagman AM. Deficit and nondeficit forms of schizophrenia: the concept. Am J Psychiatry 1988;145:578-83.

12 Kirkpatrick B, Mucci A, Galderisi S. Primary, enduring negative symptoms: an update on research. Schizophr Bull 2017;43:730-6.

13 Tek C, Kirkpatrick B, Buchanan RW. A five-year followup study of deficit and nondeficit schizophrenia. Schizophr Res 2001;49:253-60.

14 Kirkpatrick B, Buchanan RW, Ross DE, et al. A separate disease within the syndrome of schizophrenia. Arch Gen Psychiatry 2001;58:165-71.

15 Galderisi S, Merlotti E, Mucci A. Neurobiological background of negative symptoms. Eur Arch Psychiatry Clin Neurosci 2015;265:543-58.

16 Kitis O, Ozalay O, Zengin EB, et al. Reduced left uncinate fasciculus fractional anisotropy in deficit schizophrenia but not in non-deficit schizophrenia. Psychiatry Clin Neurosci 2012;66:34-43.

17 Voineskos AN, Foussias G, Lerch J, et al. Neuroimaging evidence for the deficit subtype of schizophrenia. JAMA Psychiatry 2013;70:472.

18 Lei W, Li N, Deng W, et al. White matter alterations in first episode treatment-naïve patients with deficit schizophrenia: a combined VBM and DTI study. Sci Rep 2015;5.

19 Tan AS, Chew QH, Sim K. Cerebral white matter changes in deficit and non-deficit subtypes of schizophrenia. J Neural Transm 2020;127:1073-9.

20 Spalletta G, De Rossi P, Piras F, et al. Brain white matter microstructure in deficit and non-deficit subtypes of schizophrenia. Psychiatry Res 2015;231:252-61.

21 Joshi SH, Narr KL, Philips OR, et al. Statistical shape analysis of the corpus callosum in schizophrenia. Neuroimage 2013;64:547-59.

22 Ercan I, Ocakoglu G, Sigirli D. Statistical shape analysis and usage in medical sciences. Türk Klinikleri J Biostat 2012;4:27-35.

23 Rohlf FJ. On the use of shape spaces to compare morphometric methods. Hystrix It J Mamm 2000;11.

24 Rohlf FJ. Shape statistics: Procrustes superimpositions and tangent spaces. J Classif 1999;16:197-223.

25 Slice DE. Geometric morphometrics. Annu Rev Anthropol 2007;36:261-81.
26 Kirkpatrick B, Buchanan RW, McKenney PD, et al. The schedule for the deficit syndrome: an instrument for research in schizophrenia. Psychiatry Res 1989;30:119-23.

27 Kay SR, Fiszbein A, Opler LA. The positive and negative syndrome scale (PANSS) for schizophrenia. Schizophr Bull 1987;13:261-76.

28 Kostakoglu AE, Batur S, Tiryaki A. Reliability and validity of the Turkish version of the Positive and Negative Syndrome Scale (PANSS). Turkish Journal of Psychology 1999;14:23-32.

29 First MB, Pincus HA. The DSM-IV text revision: rationale and potential impact on clinical practice. Psychiatr Serv 2002;53:288-92.

30 Sigirli D, Ercan I, Ozdemir ST, et al. Shape analysis of the corpus callosum and cerebellum in female MS patients with different clinical phenotypes. Anat Rec 2012;295:1202-11.

31 Ercan I, Ocakoglu G, Guney I. Adaptation of generalizability theory for inter-rater reliability for landmark localization. Int J Tomogr Simul 2008;9:51-8.

32 Dimitrov DM. Chap. 3, Reliability. In: Erford BT, ed. Assessment for counselors. 2 edn. Boston: Houghton-Mifflin/Lahaska Press, 2006: 99-122.

33 Lui S, Yao L, Xiao Y, et al. Resting-State brain function in schizophrenia and psychotic bipolar probands and their first-degree relatives. Psychol Med 2015;45:97-108.

34 Zhang W, Lei D, Keedy SK, et al. Brain gray matter network organization in psychotic disorders. Neuropsychopharmacology 2020;45:666-74.

35 Pettersson-Yeo W, Allen P, Benetti S, et al. Dysconnectivity in schizophrenia: where are we now? Neurosci Biobehav Rev 2011;35:1110-24.

36 Bassett DS, Nelson BG, Mueller BA, et al. Altered resting state complexity in schizophrenia. Neuroimage 2012;59:2196-207.

37 Catani M, Thiebaut de Schotten M. Atlas of human brain connections. Oxford University Press, 2013.

38 Park H-J, Kim JJ, Lee S-K, et al. Corpus callosal connection mapping using cortical gray matter parcellation and DT-MRI. Hum Brain Mapp 2008;29:503-16.

39 Kennedy KM, Raz N. Aging white matter and cognition: differential effects of regional variations in diffusion properties on memory, executive functions, and speed. Neuropsychologia 2009;47:916-27.

40 Ortiz-Gil J, Pomarol-Clotet E, Salvador R, et al. Neural correlates of cognitive impairment in schizophrenia. British Journal of Psychiatry 2011;199:202-10.

41 Friston KJ. The disconnection hypothesis. Schizophr Res 1998;30:115-25.

$42 \mathrm{Kim} \mathrm{H}$, Shon S-H, Joo SW, et al. Gray matter microstructural abnormalities and working memory deficits in individuals with schizophrenia. Psychiatry Investig 2019;16:234-43.

43 Fischer BA, Keller WR, Arango C, et al. Cortical structural abnormalities in deficit versus nondeficit schizophrenia. Schizophr Res 2012;136:51-4.

44 Turetsky B, Cowell PE, Gur RC, et al. Frontal and temporal lobe brain volumes in schizophrenia. Relationship to symptoms and clinical subtype. Arch Gen Psychiatry 1995;52:1061-70.

45 Sigmundsson T, Suckling J, Maier M, et al. Structural abnormalities in frontal, temporal, and limbic regions and interconnecting white matter tracts in schizophrenic patients with prominent negative symptoms. Am J Psychiatry 2001;158:234-43.

46 Cascella NG, Fieldstone SC, Rao VA, et al. Gray-matter abnormalities in deficit schizophrenia. Schizophr Res 2010;120:63-70.

47 Tao B, Xiao Y, Yang B, et al. Morphological alterations of the corpus callosum in antipsychotic-naive first-episode schizophrenia before and 1-year after treatment. Schizophr Res 2021;231:115-21.

48 Sigirli D, Gunes A, Turan Ozdemir S, et al. Statistical shape analysis of corpus callosum in restless leg syndrome. Neurol Res 2020;42:760-6.

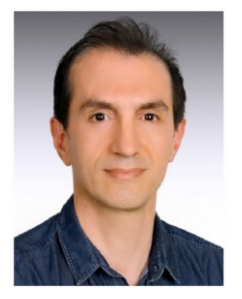

Dr Yaşar Türk obtained a medical degree from Tehran's Shahid Beheshti Medical School in Iran in 1995 and his radiology specialty from the Medical School of Istanbul University in Turkey in 2006. He completed a fellowship in neuro-interventional radiology at Zurich University Hospital. From 2017 to 2020, he worked as an assistant professor at the Radiology Department of Tekirdag Namik Kemal University in Turkey. He has been an assistant professor at the Zonguldak Bulent Ecevit University Hospital's Radiology Department in Kozlu, Zonguldak, Turkey, since 2020. He is board-certified in neuroradiology by the European Neuroradiology Board. He is presently doing DTI studies on paediatric Down syndrome and paediatric obesity as part of his resident thesis programme. 